\title{
Emphysematous cystitis complicated by liver abscess
}

Hong Kong Med J 2021;27:457.e1-2
https://doi.org/10.12809/hkmj209179

In July 2019, a 54-year-old woman with type 2 diabetes mellitus and suboptimal drug compliance with metformin and gliclazide presented to the emergency department complaining of abdominal pain, progressive weakness, and confusion. Her body temperature was $39.1^{\circ} \mathrm{C}$ and blood tests revealed serum glucose $29.1 \mathrm{mmol} / \mathrm{L}$; haemoglobin A1c $10.4 \%$; C-reactive protein $224.3 \mathrm{mg} / \mathrm{L}$; and white cell count $11.9 \times 10^{9} / \mathrm{L}$ with $92.8 \%$ segmented neutrophils; and serum creatinine $437.4 \mathrm{mmol} / \mathrm{L}$. Computed tomography scan of the abdomen and pelvis without contrast revealed a cystic gas-producing liver lesion and diffuse intramural gas dissecting the urinary bladder wall with extension to the left kidney (Fig). A diagnosis was made of acute emphysematous cystitis complicated by liver abscess.

Percutaneous drainage of the liver abscess was performed under ultrasound guidance. Klebsiella pneumoniae, sensitive to meropenem, was cultured
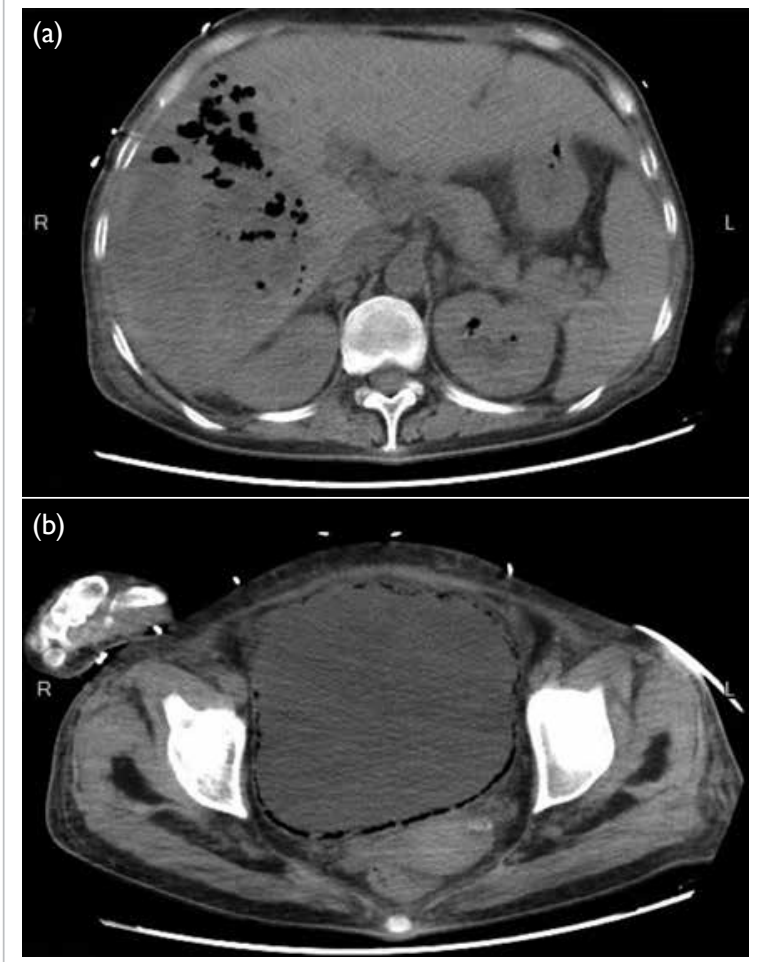

FIG. Computed tomography of the abdomen and pelvis. The patient had a gas-producing liver lesion (a) and diffuse intramural gas dissecting the urinary bladder wall (b) with extension to the left kidney from the urine and drained pus. The patient was transferred to the intensive care unit and treated with resuscitation, glycaemic control, and broadspectrum antibiotics with intravenous meropenem. Meropenem was continued for the initial 7 days and then changed to ceftazidime plus amikacin according to susceptibility testing results. Her symptoms subsided and blood parameters improved gradually. She made an uneventful recovery and was discharged from the hospital on day 20.

Emphysematous cystitis is an uncommon type of infection characterised by gas collections within the bladder wall and lumen. It is usually caused by gas-producing pathogens such as Escherichia coli and Klebsiella pneumoniae. ${ }^{1}$ Predisposing factors are diabetes, female sex, obstructive uropathy and possibly immunosuppression. Gas is believed to be produced by fermentation of albumin or glucose by the infecting organisms. Emphysematous cystitis combined with liver abscess is rare. The mechanisms of emphysematous cystitis are not well understood. Some reports emphasise that pathogenesis may be related to hematogenous transmission from the liver abscess. ${ }^{2,3}$ Immunosuppression related to poorly controlled diabetes is another contributing factor.

Clinical presentation of emphysematous cystitis varies. Our patient presented with systemic manifestations of septic shock and severe upper abdominal pain secondary to the liver abscess. She reported no lower urinary tract symptoms. Computed tomography is the best diagnostic modality with its high sensitivity when assessing the extent of gas patterns. Appropriate antibiotic therapy, correction of the underlying disorder, and adequate drainage is the recommended treatment.

\section{Author contributions}

Concept or design: Both authors.

Acquisition of data: L Zeng.

Analysis or interpretation of data: L Zeng.

Drafting of the manuscript: L Zeng.

Critical revision of the manuscript for important intellectual content: Q Wang.

Both authors had full access to the data, contributed to the study, approved the final version for publication, and take responsibility for its accuracy and integrity.

\section{Conflicts of interest}

The authors declared no potential conflicts of interest. 


\section{Funding/support}

This study received no specific grant from any funding agency in the public, commercial, or not-for-profit sectors.

\section{Ethics approval}

This study was approved by the Hospital of Chengdu University of Traditional Chinese Medicine Research Ethics Committee. Informed consent was obtained from the patient.

${ }^{1}$ L Zeng, MD

${ }^{2} \mathrm{Q}$ Wang ${ }^{*}, \mathrm{MD}$

${ }^{1}$ Department of Radiology, Hospital of Chengdu University of Traditional
Chinese Medicine, Sichuan, China

2 Department of Ultrasound, Hospital of Chengdu University of Traditional Chinese Medicine, Sichuan, China

* Corresponding author: 444028177@qq.com

\section{References}

1. Amano M, Shimizu T. Emphysematous cystitis: a review of the literature. Intern Med 2014;53:79-82.

2. Lai CC. Concomitant emphysematous cystitis and liver abscess. Korean J Intern Med 2018;33:839-40.

3. Su YC, Chen CC. Emphysematous cystitis complicating Klebsiella pneumoniae liver abscess. Am J Emerg Med 2006,24:256-7. 